\title{
Pulmonary Hypertension Associated with Bronchopulmonary Dysplasia: A Review
}

\author{
Nidhy Varghese, $M D^{1}$, and Danielle Rios, $\mathrm{MD}, \mathrm{MS}^{2}$
}

Early pulmonary vascular disease (PVD) and established pulmonary hypertension (PH) are common associations of bronchopulmonary dysplasia (BPD). Diagnosis of PH is often made by echocardiography because of technical and logistic difficulties with the neonatal population. Optimization of respiratory support is the focus of treatment, however, medical therapies are being used with increased frequency. The prognosis for $\mathrm{PH}$ associated with BPD (PH-BPD) is tied to the child's respiratory status. PH is associated with increased risk of death in this population, so active screening and treatment is recommended to minimize morbidity and mortality. In this review, we discuss the pathophysiology of PH in infants with BPD, evaluate the current understanding of screening, diagnosis, and follow-up evaluation; describe comorbid conditions; and provide a framework for targeted physiology-based management strategies.

Keywords: pulmonary hypertension, bronchopulmonary dysplasia, prematurity, sildenafil, newborns, mechanical ventilation, pulmonary vascular disease, pulmonary vein stenosis

\section{Introduction}

$\mathrm{A}$ DVANCES IN PERINATAL intensive care have improved survival rates for extremely low-gestational-age neonates. There is now an increased recognition that pulmonary vascular disease (PVD) and its most severe form, pulmonary hypertension $(\mathrm{PH})$, are commonly associated with chronic lung diseases of prematurity, specifically bronchopulmonary dysplasia (BPD). The impact of both of these disease processes on airway and vascular development are striking throughout the neonatal period. The consequences of lung injury and halted pulmonary and vascular development often continue throughout early infancy due to exposure to mechanical ventilation, oxygen administration, and fluid shifts. BPD is the consequence of this continued need for support and its classification is based on the degree of oxygen requirement and severity of respiratory disease. ${ }^{1}$

Classically, BPD is characterized by diffuse airway damage, smooth muscle hypertrophy, inflammatory cell infiltration, and parenchymal fibrosis. BPD in the modern era, known as the "new BPD," is due to injury at an earlier developmental age arresting alveolar development and impairing vascular development. ${ }^{2,3}$ Despite advances in care,
BPD rates in premature infants have remained consistently high over the past several decades. ${ }^{4}$

Early PVD and established PH are common associations of BPD. ${ }^{5}$ BPD and PH share similar risk factors and overlapping symptoms. ${ }^{6}$ Impairments in vascular development and ongoing injury from postnatal medical interventions, which are involved in the development of BPD, may also lead to elevated pulmonary vascular resistance (PVR) and a diagnosis of $\mathrm{PH}$. $\mathrm{PH}$ is a severe complication of BPD with high chronic morbidity, including poorer neurodevelopmental outcmes. ${ }^{7-10}$ Mortality rates in the $\mathrm{PH}$ associated with BPD (PH-BPD) population are reported as being 4 times higher than in the unaffected BPD population. ${ }^{8,11,12}$

\section{Definitions, Prevalence, and Risk Factors}

BPD is defined as the continued need for oxygen supplementation at 36 weeks postmenstrual age (PMA) in an infant born at $<32$ weeks gestational age. Precapillary $\mathrm{PH}$ is defined by right heart catheterization data, including mean pulmonary arterial pressures $>20 \mathrm{mmHg}$, pulmonary arterial wedge pressure $<15 \mathrm{mmHg}$, and indexed pulmonary vascular resistance $(\mathrm{PVRi})>3 \mathrm{WU}^{*} \mathrm{~m}^{2}$ in children over 3 months

Departments of ${ }^{1}$ Pediatric Pulmonology and ${ }^{2}$ Neonatology, Baylor College of Medicine, Texas Children's Hospital, Houston, Texas.

(C) Nidhy Varghese and Danielle Rios 2019; Published by Mary Ann Liebert, Inc. This Open Access article is distributed under the terms of the Creative Commons Attribution Noncommercial License (http://creativecommons.org/licenses/by-nc/4.0/) which permits any noncommercial use, distribution, and reproduction in any medium, provided the original author(s) and the source are cited. 
of age. ${ }^{13,14}$ This PH definition is adapted from adult diagnostic criteria to include PVRi for more meaningful pediatric interpretation. A term infant should reach expected adult values of PVRi by 2-3 months of age. ${ }^{13,15}$ However, the time for transition in the preterm infant is unclear.

The incidence of PH-BPD in this population is difficult to specify. Screening criteria and methods of screening are inconsistent in the published literature, making clear delineations difficult. The timing of screening also affects diagnosis. These inconsistencies are reflected in the reported prevalence of PH-BPD. Estimates vary widely in the literature, from $10 \%$ to $60 \%,{ }^{15}$ depending on study design and definitions employed. Recent PH guidelines released by the American Heart Association in conjunction with the American Thoracic Society address the lack of standardized criteria and recommend echocardiography screening of all PH-BPD infants with moderate-to-severe BPD at 36 weeks gestational age. ${ }^{16,17}$ This recommendation was echoed and specifically discussed by the Pediatric Pulmonary Hypertension Network with further details on the expectant management of the infant with PH-BPD. ${ }^{18}$

Prenatal risk factors for PH-BPD include preeclampsia, intrauterine growth restriction (IUGR), prematurity, oligohydramnios, maternal or amniotic infection, and genetic abnormalities. Postnatal risk factors are alveolar hypoxia, positive pressure ventilation, pulmonary infection, longterm corticosteroid exposure, left ventricular dysfunction, and left-to-right intracardiac shunts. ${ }^{19,20}$ In addition, early echocardiographic evidence of PVD has been identified as a risk factor for subsequent development of PH-BPD by 36 weeks PMA. ${ }^{5}$

PH-BPD differs from persistent pulmonary hypertension of the newborn (PPHN) in that the latter is a failed circulatory transition from prenatal to extrauterine life. The persistent elevation in PVR is typically in response to an insult preventing the expected transition (ie, sepsis, meconium aspiration, perinatal asphyxia), but may be idiopathic as well. ${ }^{21,22}$ PPHN is generally an acute, transient condition, which presents soon after birth. However, in some cases, for reasons not clearly understood, $\mathrm{PH}$ can persist and may predispose to $\mathrm{PH}$ later in life. ${ }^{13}$

PPHN is classified in Group 1.7 of the WHO classification, revised at the sixth World Symposium on Pulmonary Hypertension; PH-BPD is Group 3.5, falling in the category of developmental lung disorders. ${ }^{13}$ Due to the differences in pathogenesis and natural history, PH-BPD and PPHN are not interchangeable terms. It is important to correctly identify the etiology of elevated pulmonary arterial pressure because the focus of disease treatment will vary; PPHN is concerned with minimizing alveolar hypoxia, whereas the goals of targeted $\mathrm{PH}$ pharmacotherapy are to protect the right ventricle (RV) and reduce risks for dysfunction. ${ }^{15}$

\section{Pathophysiology and Symptoms}

Lung development occurs in 5 stages: embryonic, pseudoglandular, canalicular, saccular, and alveolar. ${ }^{23}$ Alveolar capillary development is largely immature until later stages of lung development, around 26 weeks' gestation. Perturbations to development in utero, such as maternal hypertensive diseases of pregnancy, poor fetal growth, and genetic abnormalities, can have negative consequences on prenatal lung development. Premature birth, coupled with acquired injury to the developing lung, contributes to markedly abnormal alveologenesis and associated vasculogenesis. ${ }^{20}$ Injury may result from the effects of hyperoxia, mechanical ventilation (volutrauma, barotrauma, atelectotrauma), hypercarbia, infection, tissue hypoxia, heart dysfunction, and intracardiac shunts.

The "old" BPD first described by Northway et al. ${ }^{24}$ in 1967 was characterized by diffuse fibroproliferative changes, reflecting effects of mechanical ventilation on the premature lung. However, in this era of extremely low-gestational-age premature infants, the "new" BPD is marked by alveolar and vascular simplification and remodeling. Abnormal gas exchange, a hallmark feature of BPD, results from abnormally thickened and fewer alveolar septae and a reduction in alveolar capillary surface area. The capillaries of the pulmonary vascular bed are found within these septae. Increased diffusion distance negatively impacts gaseous exchange, resulting in hypercapnia and hypoxemia. Mechanical ventilation and oxygen supplementation are utilized to mitigate abnormal gas exchange. However, these interventions often further contribute to lung injury. Hyperoxia creates reactive oxygen species, which can inhibit the innate factors necessary for proper angiogenesis [vascular endothelial growth factor (VEGF) and hypoxia-induced factor]. ${ }^{25}$

The end result of these pathologic changes is $\mathrm{PH}$ : a pulmonary vascular system with marked increase in PVR. Arteries are muscularized, hypertonic, and reactive. The elevated resistance impedes forward flow of blood through the lungs, which manifests as worsening hypoxemia and right ventricular adaptation by dilation and hypertrophy. The inability of the RV to sustain this response, in addition to impaired ventriculo-ventricular interaction may result in left ventricular dysfunction, contributing to the clinical picture of right heart failure.

The PH-BPD infant presents with nonspecific symptoms, which can often be ascribed to the underlying prematurity or BPD. It is well recognized that BPD with chronic respiratory failure and PH-BPD share similar risk factors and overlapping symptoms, making differentiation challenging. ${ }^{6,26}$ The PH-BPD infant may require chronic oxygen supplementation, but nevertheless may experience frequent episodes of hypoxemia and bradycardic events. Despite periods of clinical improvement, respiratory deterioration leading to escalation of respiratory support is common. In addition, the PH-BPD infant may tolerate handling poorly, requiring heavy sedation and comfort interventions. With continued exposure to elevated PVR, the RV may also fail leading to uncoupling from its afterload. RV dysfunction may manifest as hepatomegaly, edema, or poor growth, often with inability to successfully feed orally.

Evaluation of hypoxemia in the BPD infant may be further complicated by the presence of shunts. Atrial septal defects (ASDs), patent foramen ovale, and persistent patent ductus arteriosus (PDA) are common in these infants. Galambos et al. have recently reported the pathologic persistence of fetal vessels in severe BPD, suggesting alternative mechanisms for both tissue hypoxia and hypoxemia in this population. ${ }^{27}$ They postulate that these connecting vessels bypass gas exchange, causing zones of pulmonary hypoxia. The triggered hypoxic vasoconstriction pathway may contribute to the vascular remodeling in $\mathrm{PH}-\mathrm{BPD}^{20}$ and this phenomenon may have significant implications for hypoxemia management in the preterm infant who is not 
responsive to standard therapies. However, further study is needed to better characterize these lesions and their clinical significance.

\section{Screening, Diagnosis, and Evaluation}

\section{Clinical examination}

Specific subsets of BPD infants should be monitored closely for development of PH. This includes infants with severe clinical courses in the neonatal period, including those with history of oligohydramnios, IUGR, other risk factors for lung hypoplasia, slower-than-expected rate of clinical improvement; infants with severe BPD at 36 weeks PMA; infants with congenital heart defects that put them at increased risk of $\mathrm{PH}$; and infants with recurrent hypoxemic episodes, poor growth, or persistent pulmonary edema on chest radiograph.

On examination, the PH-BPD infant should be evaluated for abnormal heart sounds, right ventricular heave, or a hyperdynamic precordium, which are often difficult to appreciate in young infants. The auscultatory exam should be conducted to assess for inspiratory crackles or regional differences in air entry. The presence of fixed or intermittent low-pitched wheezing with distress may indicate significant airway malacia. The abdomen should be palpated to determine liver size and to assess for splenomegaly. Edema or anasarca are also important findings in the PH-BPD exam, as this may reflect changes in right atrial pressure, fluid status, or renal dysfunction.

\section{Laboratory analysis}

Laboratory evaluation may include baseline brain natriuretic peptide (BNP) or N-terminal pro-BNP, which could be useful to trend with repeat echocardiographic studies. ${ }^{28}$ Laboratory studies to assess respiratory function, including serial blood gas analysis, and comorbid conditions may also be considered in the evaluation of the PH-BPD infant.

\section{Echocardiography}

Echocardiography is the usual screening test for $\mathrm{PH}$ in adults and children and it is recommended in infants with established BPD. ${ }^{18}$ It may be prudent to screen all infants born at $<29$ weeks' gestation who require oxygen for $>28$ days of life. ${ }^{6,16}$ The echocardiogram may give direct signs of PH, such as increased tricuspid regurgitant jet (TRJ) and indirect signs, such as changes in the RV shape/size/function, changes in interventricular septal position, changes in right ventricular ejection time to pulmonary artery acceleration time ratio, and/or changes in direction of shunt flow
(Table 1). TRJ velocity for pulmonary artery pressure estimation may be unmeasurable because of inability to obtain a good envelope on echocardiography or it may not correlate well with catheterization data. Unmeasurable TRJ velocity may be especially pronounced in the presence of right ventricular failure. Therefore, it has been suggested to use a ratio of $\mathrm{RV}$ estimated pressure to systemic pressure for $\mathrm{PH}$ diagnosis instead of elevated TRJ velocity. This also takes the infant's systemic vascular resistance into account, when assessing right heart pressures. Using this schema, significant pressure elevation in the PH-BPD infant would be considered for $\mathrm{RV}$ :systemic ratio $>1: 2$, regardless of the calculated absolute numbers. ${ }^{15}$

Another approach is to use a higher TRJ velocity cutoff, such as $3 \mathrm{~m} / \mathrm{s}$, to be more sensitive for PH-BPD. ${ }^{29}$ Measurements of pulmonary regurgitation can be taken at peak velocity and at end velocity to calculate mean pulmonary artery pressure and pulmonary artery diastolic pressure, respectively. ${ }^{30}$ Other echocardiographic parameters, including tissue Doppler imaging, RV strain, tricuspid annular plane systolic excursion, pulmonary artery acceleration time, and $\mathrm{RV}$ fractional area change, are increasingly utilized to establish and monitor the diagnosis of $\mathrm{PH}^{31-34}$ Echocardiography should also be used to evaluate for "benign" shunts that may be contributing to the clinical picture or to assess for reversal of flow pattern in known shunts, indicating changes in pulmonary pressures (discussed below).

Imaging that can be helpful in evaluation include chest films to evaluate pulmonary vascular markings with heart size and computed tomography (CT) chest with angiography. Cardiac magnetic resonance imaging (MRI) is an emerging imaging tool for hemodynamic study using a noninvasive approach. ${ }^{35}$ However, this requires access to appropriate equipment and specialists with expertise in study protocol and interpretation. For the PH-BPD infant specifically, measurements may be difficult to obtain, limiting the power of the study. MRI studies may also be lengthy, require transport of the patient to other areas within the hospital and require general anesthesia.

\section{Cardiac catheterization}

Cardiac catheterization has been considered the gold standard for diagnosis of $\mathrm{PH}$ and is often used in children and adults with suspected PH. It is the only testing that obtains direct measurement of pulmonary hemodynamics and therefore can be used to confirm the presence of PH, grade severity, assess pulmonary vasoreactivity, define cardiac anatomy, and evaluate for other abnormalities (shunt lesions, systemicpulmonary collaterals, pulmonary vein irregularities), measure

Table 1. Severity of Pulmonary Hypertension Based on ECHO Indices 6

\begin{tabular}{lll}
\hline & \multicolumn{1}{c}{ Mild-moderate $\mathrm{PH}$} & \multicolumn{1}{c}{ Severe PH } \\
\hline Tricuspid regurgitant jet velocity & $2.5-3 \mathrm{~m} / \mathrm{s}$ & $>3 \mathrm{~m} / \mathrm{s}$ \\
RV hypertrophy & Mild to moderate & Moderate to severe \\
RV dilatation & Mild to moderate & Moderate to severe \\
RV function & Normal or mild dysfunction & Moderate to severe dysfunction \\
Interventricular septum & Normal position/mildly flattened in systole & Flattened or leftward bulging \\
Shunt through congenital lesions & Left-to-right & Bidirectional or right-to-left \\
RVET:PAAT & $>4$ & $>6$ \\
\hline
\end{tabular}

PAAT, pulmonary artery acceleration time; $\mathrm{PH}$, pulmonary hypertension; RV right ventricular; RVET, right ventricular ejection time. 
hemodynamics, and perform procedures if needed (stent placement, device closure of intracardiac shunt, angioplasty). Assessment of the left ventricle (LV) is important to assess for any prematurity-related LV complications and to assess for clinical improvement or worsening after initiation of pharmacotherapy.

Cardiac catheterization is, however, an invasive procedure typically requiring general anesthesia. Performance of cardiac catheterization in these patients is recommended in centers proficient with the care of the PH-BPD infant to minimize complications. Serious complication rates are low in experienced settings, cited as $1 \%-3 \% .^{13,15}$ Clinical stability for transport, intubation, anesthesia, along with size limitations may affect candidacy and complication rates. Cardiac catheterization should be considered if/when there $\operatorname{are}^{16}$ :

- persistent signs of severe cardiorespiratory disease or clinical deterioration not directly related to airway disease or acute infection

- significant PH despite optimal management of lung disease and associated morbidities

- long-term chronic PH therapy is anticipated

- there is the presence of unexplained, recurrent pulmonary edema

- shunt lesions, such as ASD, VSD, PDA

- concerns for pulmonary vein stenoses

\section{Comorbid Complications}

Comorbid complications are not uncommon in the $\mathrm{PH}$ BPD infant, often a consequence of extreme prematurity on developing organs. Cardiac shunts and pulmonary vein stenosis (PVS) are particularly notable comorbidities, which may significantly affect the course of $\mathrm{PH}$ progression in the BPD infant. ${ }^{16}$

\section{Left-to-Right Cardiac Shunts}

Left-to-right intracardiac shunts, such as an ASD, have traditionally been considered benign conditions in pediatric populations. However, this dogma derives from populations normal lung development. There is emerging evidence that for populations with chronic lung disease, such as BPD, any increase in pulmonary circulation can be significantly detrimental. Pulmonary overcirculation, which is difficult to measure by echocardiography, can decrease lung compliance, increase the work of breathing, and promote further vascular remodeling., ${ }^{2,36}$ Due to the cardiorespiratory impacts associated with large ASDs, these infants may exhibit poor somatic growth, an inability to tolerate weaning of oxygen supplementation, and have the need for an extended duration of respiratory support.

PDA and systemic-to-pulmonary collaterals may also contribute to increased pulmonary blood flow (Fig. 1). Even a small PDA by echocardiogram can significantly increase the ratio of pulmonary to systemic circulation (Qp:Qs), which may be poorly tolerated in a compromised lung vascular system. ${ }^{37}$ Left-to-right shunt assessment should occur with each echocardiogram. Attention should be paid to the size of the defect and direction of blood flow (ie, leftto-right, bidirectional, right-to-left), atrial and/or ventricular dilation, and diastolic septal flattening. In addition, serial chest radiographs are useful to assess for lung aeration and pulmonary edema. Diuretics and fluid restriction treatment strategies are often employed if pulmonary edema is present. Invasive evaluation and/or closure of shunts should be considered for diuretic-dependent etiologies of increased pulmonary blood flow with or without persistent inspiratory crackles on exam, poor growth, feeding intolerance, and/or inability to wean respiratory support over time.

Left-to-right shunt closure in the BPD population can be beneficial and is supported by published reports of improved growth and decreased respiratory support. ${ }^{2}$ At the time of closure, it may be discovered that PVR is actually low and the increased pressure estimate by echocardiography is merely a reflection of shunt-related increase in pulmonary artery flow. Transcutaneous closure of PDA has been reported in infants $<1,000 \mathrm{~g} .{ }^{38,39}$

However, caution should be exercised with shunt closure in the PH-BPD infant. In the infant with severe PH-BPD, the ASD or PDA may serve as a "pop-off", to decompress the $\mathrm{RV}$ in episodes of spiking pulmonary artery pressures. Echocardiography may show the shunt to be largely rightto-left or bidirectional at rest; or it may show left-to-right flow at rest, which reverses during crisis. Therefore, ASD or PDA closure in the setting of elevated PVR is often not recommended. In fact, in high PVR conditions, the ASD may be enlarged by balloon atrial septostomy. Prostaglandin E1 to maintain ductal patency can be considered or the PDA may be stented to ensure patency if the degree of $\mathrm{PH}$ is particularly severe. Subsequently after clinical improvement, these shunts can be closed by device during heart catheterization or through surgery. In recent years, cardiac catheterization is an increasingly desirable method of closure since, in addition to intervention, hemodynamics can be assessed.

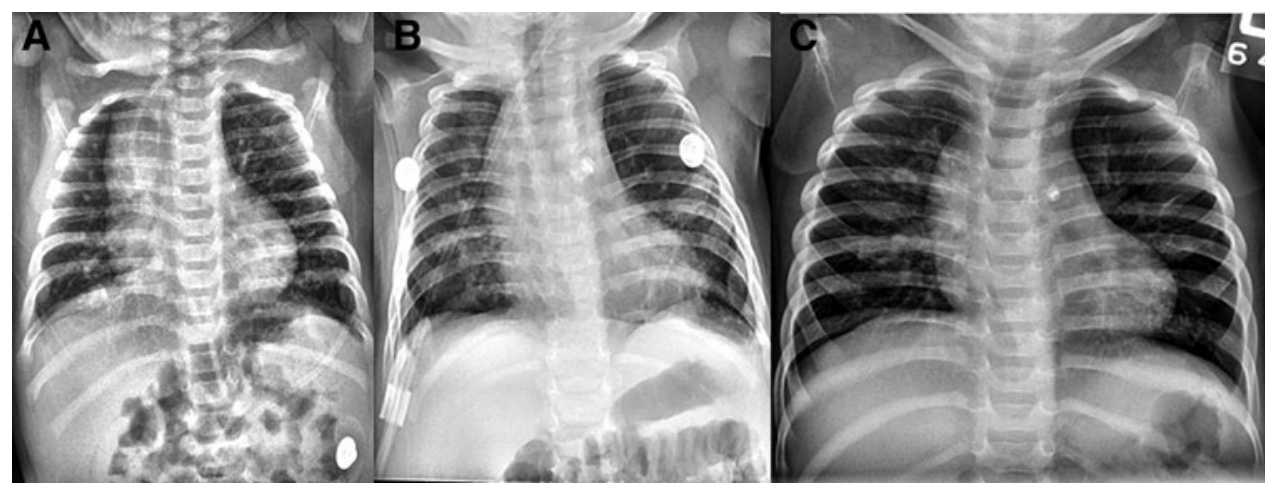

FIG. 1. Former 28-week infant with BPD and PDA (A) at 3 months of age (B) after PDA ligation $(\mathbf{C})$ at 1 year of age. BPD, bronchopulmonary dysplasia; PDA, patent ductus arteriosus. 


\section{Pulmonary Vein Stenosis}

PVS is a rare condition; however, it has been recognized as an increasingly prevalent complication for the premature population with BPD. ${ }^{40}$ PVS is marked by intimal and medial fibromuscular or fibromyxoid proliferation causing narrowing of the intra- and/or extrapulmonary pulmonary veins up to the venoatrial junction (Fig. 2). ${ }^{41}$ This will result in some degree of pulmonary venous congestion, interstitial edema, and upstream elevation of pulmonary artery pressure. The arterial involvement makes PVS a significantly difficult condition to treat and assess because of the pre- and postcapillary PH. Pulmonary vasodilation may result in capillary flooding and pulmonary edema.

PVS may be congenital or acquired. Observational studies in infants with BPD with serial echocardiograms show the first detection of PVS in late infancy/toddler stage, indicating that this condition is likely acquired and develops over the course of several months. The etiology of PVS in the BPD population is unclear and generally thought to be idiopathic. However, causes related to the prematurity itself have been implicated. These factors include stressors within the underdeveloped premature heart, such as increased left ventricular loading conditions, which could adversely affect postnatal pulmonary vein development. Prolonged mechanical ventilation has also been evoked as a risk factor for PVS development in BPD, perhaps through stimulation of inflammation and VEGF pathways. ${ }^{11,40}$ An association with necrotizing enterocolitis has also been suggested. ${ }^{41}$

The natural history of PVS is extremely unpredictable. ${ }^{40}$ PVS is often progressive and may "spread," with unaffected veins becoming affected over time. The degree of involvement may also vary, from mild stenosis to complete atresia of the vein. Echocardiography will diagnose the majority of PVS cases, however, the condition may also be diagnosed by contrast CT scan with angiography, angiography during cardiac catheterization, and cardiac MRI. ${ }^{42}$

PVS should be considered in the preterm infant with increasing oxygen requirement and worsening uni- or multilobar haziness on chest radiography. Echocardiography typically suggests worsening PH. Specific attention should be paid to the pulmonary veins during echocardiography in this patient population as they may not be interrogated on routine studies. Turbulent flow with increasing gradient $>5 \mathrm{mmHg}$ is concerning for PVS. ${ }^{43}$ In cases of complete pulmonary vein atresia, there will be no flow noted by Doppler on echocardiogram and CT scan of the chest with angiography should be pursued for confirmation of the diagnosis.
Treatment for PVS is challenging and often unsuccessful long term. PH-targeted therapy may be initiated for the precapillary component of $\mathrm{PH}$. The risk for pulmonary edema may be minimized if this therapy is initiated very slowly with careful concomitant use of diuretics. Catheterization-based interventional therapy for PVS can be done through balloon angioplasty $+/-$ stent placement. Medical antiproliferative therapy (ie, sirolimus) may be trialed alone or in combination with specially designed drug-eluting stents. However, the use of specific pharmacologic agents has not been described in prematurely born infants. Surgical intervention through resection of the stenotic segments of pulmonary veins with reimplantation or marsupialization of the affected vein(s) using a sutureless technique has been reported. ${ }^{44}$ Finally, for progressive PVS, lung transplantation is an ultimate consideration, although candidacy may be affected by type of primary surgical repair (ie, sutureless technique).

The prognosis of BPD infants with PVS is significantly worse than that of BPD infants without PVS, cited to be $\sim 50 \%$ at 24 months of age. ${ }^{11}$ Prognosis is related to the number of veins involved, the extent of the stenosis (ie, long segment, complete atresia), and amenability to intervention, which reflects the degree of ongoing intimal and medial proliferation. PVS in the setting of PH-BPD may confer an extremely guarded prognosis due to disease progression and challenges in balancing treatment for the pre- and postcapillary disease.

\section{Management}

Treatment of the PH-BPD infant focuses on preserving right ventricular function by preventing progression of pulmonary vascular remodeling, minimizing further insult to the pulmonary vasculature, and optimizing respiratory status. General neonatology principles of care form the basis for much of the recommendations for PH-BPD management. In general, as infants grow and mature, new alveolar growth with attendant capillaries leads most survivors to improve with respect to both lung disease and $\mathrm{PH}$. However, some children may require use of targeted $\mathrm{PH}$ therapies to manage the disease process more long term. It should be noted though that there are no randomized controlled studies of targeted $\mathrm{PH}$ pharmacotherapy in BPD. Echocardiography should be repeated at regular intervals to monitor disease status, response to therapy, and for left ventricular diastolic dysfunction. ${ }^{18,45}$

\section{Management of chronic respiratory failure}

Successful management of respiratory failure is a crucial and potentially curative component of the management of
FIG. 2. Cardiac CT with contrast showing segmental stenosis of the LUPV as it drains into the left atrium (arrows). CT, computed tomography; LUPV, left upper pulmonary vein.

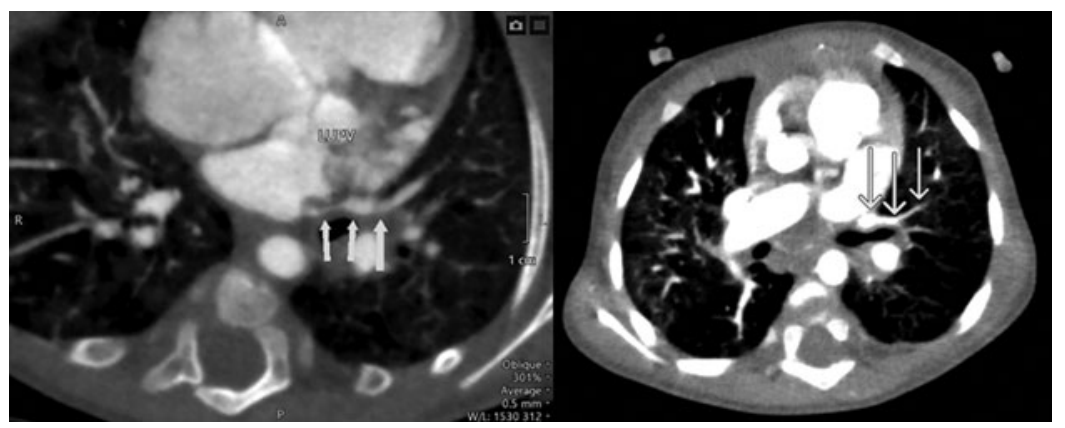


PH. Patients should be assessed for structural airway abnormalities when suspected; this may require flexible and/or rigid bronchoscopy and/or dynamic assessment of airway caliber by CT scan. Both tracheomalacia and bronchomalacia have been reported commonly in this patient population. Acute and/or chronic respiratory failure must be addressed in a timely fashion to minimize further injury to an abnormal pulmonary vascular bed.

Experts recommend using ventilator techniques which minimize hyperinflation because of the negative effects on PVR associated with high lung volumes, but which will also minimize alveolar derecruitment. Ventilatory strategies for PH-BPD build on BPD expert consensus recommendations, to minimize air trapping and avoid acidosis: respiratory rates $12-15$ breaths per minute, tidal volumes $10-12 \mathrm{~mm} / \mathrm{kg}$ of body weight, and positive end-expiratory pressures $<10 \mathrm{~cm}$ of water. ${ }^{16}$ In some instances, long-term mechanical ventilation may be necessary. ${ }^{20}$

The use of corticosteroids for prevention of BPD has been studied extensively, yet the exact regimen remains controversial. Their role in PH-BPD has not been evaluated. ${ }^{46}$ Additional morbidities, such as chronic dysphagia with aspiration, also require management to prevent further injury to fragile BPD lungs. ${ }^{16,47}$ Measures to minimize respiratory exacerbations due to respiratory viral illnesses are crucial to preserving prognosis. Local infection control policies should be enforced, along with good hand washing. Vaccinations should be routinely administered for the patient and recommended for parents, family members, the health care team, and visitors. If eligible, prophylaxis for respiratory syncytial virus should be given.

\section{Nutrition}

Optimized nutrition may be difficult in the infant with $\mathrm{PH}-$ BPD due to fluid restriction. However, strategies, such as caloric fortification and volume condensation, may be reasonable options to continue delivery of high-quality nutrition. $^{47}$ Specific nutritional strategies that focus on somatic and lung growth are beyond the scope of this review, but there is a recognized need for targeted nutritional support with supervised titration to promote optimal nutritional success. ${ }^{47}$

\section{Oxygen}

Oxygen in high concentrations may serve as a vasodilator and oxygen supplementation is typically used in BPD and PH-BPD. The goal of oxygen supplementation is to reduce or eliminate hypoxemic episodes. Arterial oxygen saturation $\left(\mathrm{SaO}_{2}\right)$ is generally measured by oximetry and is used in $\mathrm{PH}$ patients as a surrogate for alveolar oxygenation. Target $\mathrm{SaO}_{2}$ varies based on the etiology of the $\mathrm{PH}$ and coexistence of additional morbidities, such as congenital heart disease and ophthalmologic disease. In severe PVS, a slightly lower oxygen target may be desirable to control oxygen-mediated precapillary vasodilation. Hyperoxia should be minimized to reduce additional oxygen toxicity and pulmonary inflammation. Recently released guidelines recommend a target $\mathrm{SaO}_{2}$ of $92 \%-94 \%$ as sufficient to prevent hypoxemia, while not incurring additional inflammation from hyperoxia and oxygen toxicity. ${ }^{16}$

In some cases, there may be poor or absent clinical response to oxygen supplementation. An intracardiac shunt lesion or pulmonary arteriovenous malformation should be considered. Intrapulmonary bronchopulmonary anastomoses in severe BPD may also contribute to recorded hypoxemia. However, these lesions are presently undetectable by standard investigation, therefore making them a difficult clinical target. In older infants or toddlers with persistent $\mathrm{PH}$ by echocardiography, a formal polysomnogram may be required to evaluate for sleep disordered breathing. ${ }^{16}$

Diuretics may be clinically effective in the setting of right ventricular dilation and congestion. ${ }^{25}$ Therapy is titrated to effect, which may be especially important in the setting of shunt lesions. Care must be taken to maintain cardiac preload. ${ }^{13}$

Inhaled nitric oxide is generally used only in selected patients in a dose range of 1-20 parts per million; methemoglobin levels should be monitored. Inhaled nitric oxide is indicated if the partial pressure of oxygen in an arterial sample is $<100 \mathrm{mmHg}$ (while receiving $100 \%$ oxygen) or if oxygenation index is $>25$. $^{16,45}$

\section{Targeted Specific PH Pharmacotherapy}

\section{Phosphodiesterase inhibitors}

The PDE-5 (phosphodiesterase-5) inhibitors increase intracellular cGMP levels, possibly promoting alveolar growth and associated vasculogenesis. Inflammation and airway reactivity have also been shown to be reduced. This class of $\mathrm{PH}$ drugs is more selective for the pulmonary circulation, but can have systemic effects such as hypotension and gastroesophageal reflux. In 2009, Mourani et al. reported that chronic sildenafil therapy was well tolerated in PH-BPD infants. ${ }^{48}$ In neonatal animal models, sildenafil decreased lung inflammation and extravascular fibrin deposition, decreased right ventricular hypertrophy, and improved survival. ${ }^{49}$

In 2012, the Food and Drug Administration (FDA) issued a safety communication recommending against the use of Revatio (sildenafil) in children with $\mathrm{PH}$, following results from the STARTS-1 trial. ${ }^{50,51}$ The patient population included children over the age of 1 year. The statement was clarified in 2014 to encourage prescribers to consider the risk-benefit profile for informed decision making in the pediatric population. ${ }^{52}$ Despite the warnings and early public reaction, sildenafil remains the most widely used $\mathrm{PH}$ therapy in the PH-BPD population. ${ }^{53}$

\section{Endothelin receptor antagonists}

Endothelin receptor antagonists block the endothelin receptor, preventing the action of naturally occurring endothelin hormone (vasoconstriction and proliferation). Bosentan, an endothelin receptor A and receptor B antagonist, is the only $\mathrm{PH}$ medication approved by the FDA for use in pediatric $\mathrm{PH}$. Common side effects include changes in liver enzymes so monthly monitoring of hepatic function is required by the FDA. Other side effects include anemia and edema. Bosentan may be used alone or in combination with other PH therapies. ${ }^{54}$

\section{Prostacyclins}

As a group, prostacyclins have a variety of effects, including vasodilation, remodeling of pulmonary arterioles, reduction in inflammation, and decrease in platelet aggregation. 
Depending on route of administration (oral, inhaled, subcutaneous infusion, intravenous infusion), side effects may include hypotension, worsening hypoxemia, rash, feeding intolerance, diarrhea, pyrexia, and complications related to vascular line access. Prostacyclins are generally reserved for severe $\mathrm{PH}$ and are generally considered as add-on therapy for the infant with PH-BPD who is refractory to other interventions. ${ }^{16,53,55}$ Prostacyclins may also be used acutely in the immediate management of right ventricular failure or $\mathrm{PH}$ crisis. Successful use of inhaled iloprost and nebulized epoprostenol has also been reported in the literature.

\section{Prognosis}

PVD in BPD can be recognized on echocardiogram as early as the first week of life. As noted previously, the early diagnosis of PVD on echocardiography is a risk factor for both BPD and late $\mathrm{PH} .{ }^{5}$ An increased duration of respiratory support and the severity of parenchymal lung disease are also associated with significant late $\mathrm{PH}$. While $\mathrm{PH}$ is highly prevalent in infants with severe BPD, there is no clear linear relationship between severity of BPD and $\mathrm{PH}$; infants with mild or no BPD can also have evidence of increased PVR. Therefore, vigilance for this vulnerable population is advised.

PH medications have been used with success in various neonatal populations, although in uncontrolled retrospective reports. However, optimization of respiratory status remains the principal therapy for most infants with PH-BPD. In the infant population, mortality rates have been reported to be as high as $66 \%{ }^{19}$

While $\mathrm{PH}$ is a serious risk factor for increased mortality in this vulnerable group, with adequate growth, proper respiratory support, avoidance of infection, and management of comorbid conditions, the risk of death decreases over time. Few infants will have chronic $\mathrm{PH}$ requiring medical therapies past early childhood. Risk for acute PH (ie, associated with acute respiratory failure in the setting of a respiratory infection) remains but also typically decreases over time. This decrease in risk is related to de novo lung growth with concomitant vascular growth in the first years of childhood. Therefore, dependence and use of most respiratory support, such as oxygen supplementation, tracheostomy, and mechanical ventilation, typically decrease over time.

However, careful serial follow-up for this patient population is of the utmost importance. PH-related morbidity can persist past infancy and well into adulthood. ${ }^{56}$ Longitudinal and follow-up studies of BPD have revealed continuation of abnormal adaptive responses of the heart and vasculature into childhood, suggesting that there is a component of persistent vascular abnormality. ${ }^{57}$ In some BPD survivors, clinically significant $\mathrm{PH}$ may present or reoccur in early adulthood. Neurodevelopmental outcomes (cognitive, language, and motor) - and quality of life for PH-BPD infants have been reported to be significantly abnormal, with delays persisting to the early toddler years. ${ }^{10,58}$

\section{Conclusion}

Although a substantial subset of very premature infants will develop BPD, PVD and ultimately PH affect only a minority of the population during initial hospitalization and at discharge. However, premature infants with PH-BPD have persistent morbidity and higher mortality rates compared with gestation-matched controls. The respiratory status must be carefully managed to minimize hypercapnia and hypoxemia. Assessment for comorbidities, such as intracardiac left-to-right shunts, PVS, and complications of prematurity, is crucial to prevent further injury to a developing, fragile vascular system. PH-targeted therapies may be trialed in this population but care should be taken to minimize exacerbations of V/Q mismatch. Timely diagnosis combined with appropriate intervention have been suggested to improve prognosis. However, further study is needed to determine PH-BPD-specific criteria for echocardiography diagnosis and to further guide therapeutic management for this severe form of PVD.

\section{Author Disclosure Statement}

No competing financial interests exist.

\section{Funding Information}

No funding was received for this article.

\section{References}

1. Jobe AH, Bancalari E. Bronchopulmonary dysplasia. Am J Respir Crit Care Med 2001; 163:1723-1729.

2. Thomas VC, Vincent R, Raviele A, et al. Transcatheter closure of secundum atrial septal defect in infants less than 12 months of age improves symptoms of chronic lung disease. Congenit Heart Dis 2012; 7:204-211.

3. Perez VAdJ. Long-term right ventricular adaptation to postnatal hyperoxia: too much of a good thing? Am J Respir Cell Mol Biol 2017; 56:559-560.

4. Zysman-Colman Z, Tremblay GM, et al. Bronchopulmonary dysplasia-trends over three decades. Paediatr Child Health 2013; 18:86-90.

5. Mourani PM, Sontag MK, Younoszai A, et al. Early pulmonary vascular disease in preterm infants at risk for bronchopulmonary dysplasia. Am J Respir Crit Care Med 2015; 191:87-95.

6. Nagiub M, Lee S, Guglani L. Echocardiographic assessment of pulmonary hypertension in infants with bronchopulmonary dysplasia: systematic review of literature and a proposed algorithm for assessment. Echocardiography $2015 ; 32: 819-833$.

7. Nakanishi H, Uchiyama A, Kusuda S. Impact of pulmonary hypertension on neurodevelopmental outcome in preterm infants with bronchopulmonary dysplasia: a cohort study. J Perinatol 2016; 36:890-896.

8. Lagatta JM, Hysinger EB, Zaniletti I, et al. The impact of pulmonary hypertension in preterm infants with severe bronchopulmonary dysplasia through 1 year. J Pediatr 2018; 203:218-224.e3.

9. Blanca AJ, Duijts L, van Mastrigt E, et al. Right ventricular function in infants with bronchopulmonary dysplasia and pulmonary hypertension: a pilot study. Pulm Circ 2019; 9: $1-9$.

10. Choi EK, Shin SH, Kim EK, et al. Developmental outcomes of preterm infants with bronchopulmonary dysplasiaassociated pulmonary hypertension at 18-24 months of corrected age. BMC Pediatr 2019; 19:26.

11. Swier NL, Richards B, Cua CL, et al. Pulmonary vein stenosis in neonates with severe bronchopulmonary dysplasia. Am J Perinatol 2016; 33:671-677. 
12. Arjaans S, Zwart EAH, Ploegstra M-J, et al. Identification of gaps in the current knowledge on pulmonary hypertension in extremely preterm infants: a systematic review and meta-analysis. Paediatr Perinat Epidemiol 2018; 32:258267.

13. Rosenzweig EB, Abman SH, Adatia I, et al. Paediatric pulmonary arterial hypertension: updates on definition, classification, diagnostics and management. Eur Respir J 2019; 53. pii: 1801916.

14. Galiè N, McLaughlin VV, Rubin LJ, et al. An overview of the 6th world symposium on pulmonary hypertension. Eur Respir J 2019; 53. pii: 180214.

15. Keller RL. Pulmonary hypertension and pulmonary vasodilators. Clin Perinatol 2016; 43:187-202.

16. Abman SH, Hansmann G, Archer SL, et al. Pediatric pulmonary hypertension: guidelines from the American Heart Association and American Thoracic Society. Circulation 2015; 132:2037-2099.

17. Vayalthrikkovil S, Vorhies E, Stritzke A, et al. Prospective study of pulmonary hypertension in preterm infants with bronchopulmonary dysplasia. Pediatr Pulmonol 2019; 54: 171-178.

18. Krishnan U, Feinstein JA, Adatia I, et al. Evaluation and management of pulmonary hypertension in children with bronchopulmonary dysplasia. J Pediatr 2017; 188:2434.e1.

19. Khemani E, McElhinney DB, Rhein L, et al. Pulmonary artery hypertension in formerly premature infants with bronchopulmonary dysplasia: clinical features and outcomes in the surfactant era. Pediatrics 2007; 120:12601269.

20. Baker CD, Abman SH, Mourani PM. Pulmonary hypertension in preterm infants with bronchopulmonary dysplasia. Pediatr Allergy Immunol Pulmonol 2014; 27:8-16.

21. Lai M-Y, Chu S-M, Lakshminrusimha S, et al. Beyond the inhaled nitric oxide in persistent pulmonary hypertension of the newborn. Pediatr Neonatol 2018; 59:15-23.

22. Fuloria M, Aschner JL. Persistent pulmonary hypertension of the newborn. Semin Fetal Neonatal Med 2017; 22:220 226.

23. Herriges M, Morrisey EE. Lung development: orchestrating the generation and regeneration of a complex organ. Development 2014; 141:502-513.

24. Northway Jr. WH, Rosan RC, Porter DY. Pulmonary disease following respirator therapy of hyaline-membrane disease - bronchopulmonary dysplasia. N Engl J Med. 1967; 276:357-368.

25. Valencia AM, Abrantes MA, Hasan J, et al. Reactive oxygen species, biomarkers of microvascular maturation and alveolarization, and antioxidants in oxidative lung injury. React Oxyg Species (Apex) 2018; 6:373-388.

26. Neary E, Jain A. Right ventricular congestion in preterm neonates with chronic pulmonary hypertension. J Perinatol 2018; 38:1708-1710.

27. Galambos C, Sims-Lucas S, Abman SH. Histologic evidence of intrapulmonary anastomoses by three-dimensional reconstruction in severe bronchopulmonary dysplasia. Ann Am Thorac Soc 2013; 10:474-481.

28. Cuna A, Kandasamy J, Sims B. B-type natriuretic peptide and mortality in extremely low birth weight infants with pulmonary hypertension: a retrospective cohort analysis. BMC Pediatr 2014; 14:68.

29. Mourani PM, Sontag MK, Younoszai A, et al. Clinical utility of echocardiography for the diagnosis and manage- ment of pulmonary vascular disease in young children with chronic lung disease. Pediatrics 2008; 121:317-325.

30. Parasuraman S, Walker S, Loudon BL, et al. Assessment of pulmonary artery presuure by echocardiography-A comprehensive review. Int J Cardiol Heart Vasc 2016; 12: 45-51.

31. Singh GK, Levy PT, Holland MR, et al. Novel methods for assessment of right heart structure and function in pulmonary hypertension. Clin Perinatol 2012; 39:685-701.

32. Breatnach CR, Levy PT, James AT, et al. Novel echocardiography methods in the functional assessment of the newborn heart. Neonatology 2016; 110:248-260.

33. Levy PT, Patel MD, Groh G, et al. Pulmonary artery acceleration time provides a reliable estimate of invasive pulmonary hemodynamics in children. J Am Soc Echocardiogr 2016; 29:1056-1065.

34. Levy PT, Holland MR, Sekarski TJ, et al. Feasibility and reproducibility of systolic right ventricular strain measurement by speckle-tracking echocardiography in premature infants. J Am Soc Echocardiogr 2013; 26:1201-1213.

35. Groves AM, Chiesa G, Durighel G, et al. Functional cardiac MRI in preterm and term newborns. Arch Dis Child Fetal Neonatal Ed 2011; 96:F86-F91.

36. Choi EK, Jung YH, Kim H-S, et al. The impact of atrial left-to-right shunt on pulmonary hypertension in preterm infants with moderate or severe bronchopulmonary dysplasia. Pediatr Neonatol 2015; 56:317-323.

37. del Cerro MJ, Sabaté Rotés A, Cartón A, et al. Pulmonary hypertension in bronchopulmonary dysplasia: clinical findings, cardiovascular anomalies and outcomes. Pediatr Pulmonol 2014; 49:49-59.

38. Zahn EM, Nevin P, Simmons C, et al. A novel technique for transcatheter patent ductus arteriosus closure in extremely preterm infants using commercially available technology. Catheter Cardiovasc Interv 2015; 85:240-248.

39. Zahn EM, Peck D, Phillips A, et al. Transcatheter closure of patent ductus arteriosus in extremely premature newborns: early results and midterm follow-up. JACC Cardiovasc Interv 2016; 9:2429-2437.

40. Mahgoub L, Kaddoura T, Kameny AR, et al. Pulmonary vein stenosis of ex-premature infants with pulmonary hypertension and bronchopulmonary dysplasia, epidemiology, and survival from a multicenter cohort. Pediatr Pulmonol 2017; 52:1063-1070.

41. Nasr VG, Callahan R, Wichner Z, et al. Intraluminal pulmonary vein stenosis in children: a "new" lesion. Anesth Analg 2019; 129:27-40.

42. Laux D, Rocchisani M-A, Boudjemline Y, et al. Pulmonary hypertension in the preterm infant with chronic lung disease can be caused by pulmonary vein stenosis: a mustknow entity. Pediatr Cardiol 2016; 37:313-321.

43. Drossner DM, Kim DW, Maher KO, et al. Pulmonary vein stenosis: prematurity and associated conditions. Pediatrics 2008; 122:e656-e661.

44. Azakie A, Lavrsen MJ, Johnson NC, et al. Early outcomes of primary sutureless repair of the pulmonary veins. Ann Thorac Surg 2011; 92:666-672.

45. Hilgendorff A, Apitz C, Bonnet D, et al. Pulmonary hypertension associated with acute or chronic lung diseases in the preterm and term neonate and infant. The European paediatric pulmonary vascular disease network, endorsed by ISHLT and DGPK. Heart 2016; 102:ii49-ii56.

46. Onland W, De Jaegere AP, Offringa M, et al. Systemic corticosteroid regimens for prevention of bronchopu- 
monary dysplasia in preterm infants. Cochrane Database Syst Rev 2017; 1:CD010941.

47. Abman SH, Collaco JM, Shepherd EG, et al. Interdisciplinary care of children with severe bronchopulmonary dysplasia. J Pediatr 2017; 181:12-28.e1.

48. Mourani PM, Sontag MK, Ivy DD, et al. Effects of longterm sildenafil treatment for pulmonary hypertension in infants with chronic lung disease. J Pediatr 2009; 154:379_ 384.e2.

49. de Visser YP, Walther FJ, Laghmani EH, et al. Sildenafil attenuates pulmonary inflammation and fibrin deposition, mortality and right ventricular hypertrophy in neonatal hyperoxic lung injury. Respir Res 2009; 10:30.

50. FDA. (2012, 12/15/2017). FDA Drug Safety Communication: FDA recommends against use of Revatio (sildenafil) in children with pulmonary hypertension. https://www.fda .gov/Drugs/DrugSafety/ucm317123.htm\#safety (accessed December 17, 2018).

51. Barst RJ, Ivy DD, Gaitan G, et al. A randomized, doubleblind, placebo-controlled, dose-ranging study of oral sildenafil citrate in treatment-naive children with pulmonary arterial hypertension. Circulation 2012; 125:324-334.

52. FDA. (2014, 1/15/2016). FDA Drug Safety Communication: FDA clarifies Warning about Pediatric Use of Revatio (sildenafil) for Pulmonary Arterial Hypertension. https:// www.fda.gov/Drugs/DrugSafety/ucm390876.htm (accessed December 17, 2018).

53. Lakshminrusimha S, Mathew B, Leach CL. Pharmacologic strategies in neonatal pulmonary hypertension other than nitric oxide. Semin Perinatol 2016; 40:160-173.
54. Kadmon G, Schiller O, Dagan T, et al. Pulmonary hypertension specific treatment in infants with bronchopulmonary dysplasia. Pediatr Pulmonol 2017; 52:77-83.

55. McIntyre CM, Hanna BD, Rintoul N, et al. Safety of epoprostenol and treprostinil in children less than 12 months of age. Pulm Circ 2013; 3:862-869.

56. Nathan SD, Barbera JA, Gaine SP, et al. Pulmonary hypertension in chronic lung disease and hypoxia. Eur Respir J 2019; 53. pii: 1801914.

57. Koroglu OA, Yalaz M, Levent E, et al. Cardiovascular consequences of bronchopulmonary dysplasia in prematurely born preschool children. Neonatology 2013; 104: 283-289.

58. Cheong JLY, Doyle LW. An update on pulmonary and neurodevelopmental outcomes of bronchopulmonary dysplasia. Semin Perinatol 2018; 42:478-484.

Address correspondence to:

Nidhy Varghese, MD

Department of Pediatric Pulmonology

Baylor College of Medicine

Texas Children's Hospital

6701 Fannin

Suite 1040.00

Houston, TX 77030

E-mail: npvarghe@texaschildrens.org

Received for publication December 24, 2018; accepted after revision September 18, 2019. 